\title{
Brittle Behavior in Aspirin Crystals: Evidence of Spalling Fracture
}

DOI:

10.1021/acs.cgd.0c01662

\section{Document Version}

Accepted author manuscript

Link to publication record in Manchester Research Explorer

\section{Citation for published version (APA):}

Gabriele, B. P. A., Williams, C. J., Stauffer, D., Derby, B., \& Cruz-Cabeza, A. J. (2021). Brittle Behavior in Aspirin Crystals: Evidence of Spalling Fracture. Crystal Growth \& Design. https://doi.org/10.1021/acs.cgd.0c01662

\section{Published in:}

Crystal Growth \& Design

\section{Citing this paper}

Please note that where the full-text provided on Manchester Research Explorer is the Author Accepted Manuscript or Proof version this may differ from the final Published version. If citing, it is advised that you check and use the publisher's definitive version.

\section{General rights}

Copyright and moral rights for the publications made accessible in the Research Explorer are retained by the authors and/or other copyright owners and it is a condition of accessing publications that users recognise and abide by the legal requirements associated with these rights.

\section{Takedown policy}

If you believe that this document breaches copyright please refer to the University of Manchester's Takedown Procedures [http://man.ac.uk/04Y6Bo] or contact uml.scholarlycommunications@manchester.ac.uk providing relevant details, so we can investigate your claim.

\section{OPEN ACCESS}




\title{
Brittle Behaviour in Aspirin Crystals: Evidence of Spalling Fracture
}

\author{
Benjamin P. A. Gabriele ${ }^{\mathrm{a}}$, Craig J. Williams ${ }^{\mathrm{b}}$, Douglas Stauffer ${ }^{\mathrm{c}}$, Brian Derby ${ }^{\mathrm{b}}$, \\ Aurora J. Cruz-Cabeza ${ }^{\text {a* }}$ \\ ${ }^{a}$ Department of Chemical Engineering and Analytical Science, University of Manchester, UK. \\ ${ }^{b}$ Department of Materials, University of Manchester, UK. \\ ${ }^{\mathrm{c}}$ Bruker Nano Surfaces, Bruker Inc., Eden Prairie, MN, USA. \\ *Email: aurora.cruzcabeza@manchester.ac.uk
}

\begin{abstract}
Single crystals of aspirin form I were cleaved and indented on their dominant face. Upon inspection, it was possible to observe strongly anisotropic shallow lateral cracks due to the extreme low surface roughness after cleavage. Atomic Force Microscopy (AFM) imaging showed spalling fractures nucleating from the indent corners, forming terraces with a height of one or two interplanar spacings $d_{100}$. The formation of such spalling fractures in aspirin was rationalised using basic calculations of attachment energies, showing how (100) layers are poorly bonded when compared to their relatively higher intralayer bonding. An attempt at explaining the preferential propagation of these fractures along the [010] direction is discussed.
\end{abstract}

Key words: aspirin, nanoindentation, spalling fracture

Many everyday products contain molecular crystals, including foodstuffs, paints and, almost ubiquitously, in pharmaceuticals. Processing those into products requires multiple and diverse processes such as crystallisation, filtration, drying, milling, granulation and tableting. The mechanical properties of crystals may affect how they behave during some of such processes. It is often necessary to devise complex formulations to correct such behaviour. However, it can be difficult to determine the mechanical behaviour of molecular crystals accurately because of difficulties in preparing sufficiently large specimens for testing. Interest in the study of mechanical properties of crystalline materials has resurged in recent years thanks to new techniques, such as nanoindentation, which can produce reproducible data from small sample volumes. There are extensive nanoindentation studies on single crystals of metals, alloys, ceramics and many other types of inorganic materials available in the literature but more rarely on molecular crystals. Recent pioneering work by Desiraju and Rammamurty ${ }^{1}$ showed how nanoindentation can be applied to molecular single crystal specimens to measure their mechanical properties. Hence, in the past decade, there have been significantly more studies on the impact of polymorphism, anisotropy and co-crystallisation on mechanical properties of molecular single crystals. ${ }^{2}$

Characterising mechanisms of plastic deformation at the molecular level is key to understanding and controlling a material's mechanical behaviour. Current opinion is that plastic deformation processes undergone by crystalline molecular materials are similar to those observed in inorganic crystals and metals. The work of Reddy et al. has shed some light as to 
the structure-property relationships of molecular crystals deforming by shearing, bending and cracking and noted the utility of nanoindentation in characterizing their mechanical properties. ${ }^{3}$ Understanding, designing and controlling such behaviour can be very powerful. For example, crystals capable of bending without fracture have been shown to have improved tabletability, a property that can be engineered through polymorph control or co-crystallisation. ${ }^{4,5}$ Still, most molecular crystals are brittle, ${ }^{6}$ a behaviour often observed upon nanoindentation through the appearance of radial cracks which propagate from the indents and mostly along specific crystal orientations, due to the high degree of anisotropy found in these materials. For example, cracks have been reported in single crystals of form I aspirin which, upon nanoindentation, propagate along the [010] direction. ${ }^{7}$ In this context, we report here on a type of brittle fracture, spalling fracture or lateral cracking, which, to the best of our knowledge, has been previously unreported in molecular crystals.

Using our method detailed elsewhere, ${ }^{8}$ we have indented single crystals of aspirin form I (displacement controlled-mode) on their dominant face, aspirin-I-(100), using a Hysitron TI 950 TriboIndenter equipped with a diamond Berkovich tip (Bruker, Minneapolis MN, USA). Prior to the indentation experiments, (100) surfaces of single crystals of aspirin-I were cleaved using adhesive tape. This resulted in the generation of extremely smooth aspirin-I-(100) surfaces: the surface roughness values around the selected areas for indentation ranged from $0.45 \mathrm{~nm}$ to $2.02 \mathrm{~nm}$. This was here important in enabling precise imaging of surface features after nanoindentation. All load-depth curves obtained from nanoindentation on aspirin-I-(100) faces (Figure 1a) show sudden bursts of deformation upon loading the tip into the material (commonly referred to as "pop-ins"). "Pop-ins", enhanced downward displacements with no load increment, are associated with the onset of plastic flow or cracking ${ }^{9}$. These events are complex and whilst their intensities have been previously correlated with relevant crystallographic directions ${ }^{7}$, we have not observed such correlations. Our discrepancy with previous observations may be due to the differences in the experimental conditions used (displacement-controlled, different maximum load used...) and also to the complexity of the events associated with the pop-ins. More interestingly, we observed "pop-ins" upon unloading in several of our load-depth curves (Figure 1). "Pop-ins" are commonly reported upon loading and "pop-outs" (the inverse of a pop-in showing an upward displacement with no decrease in load) upon unloading which are a sign of polymorphic pressure-induced phase transitions. "Pop-ins" upon unloading, however, have rarely been reported. A "pop-in" upon unloading (Figure 1b) indicates a sudden displacement to a greater depth as the tip is nominally being withdrawn from the material during the unload segment. Discontinuous events during unloading have been reported to be associated with lateral cracks, ${ }^{10,11}$ whose formation is attributed to the tensile stress created by the elastic recovery upon unloading and interactions with the surrounding plastic zone that formed during indentation. ${ }^{12}$ 
a)

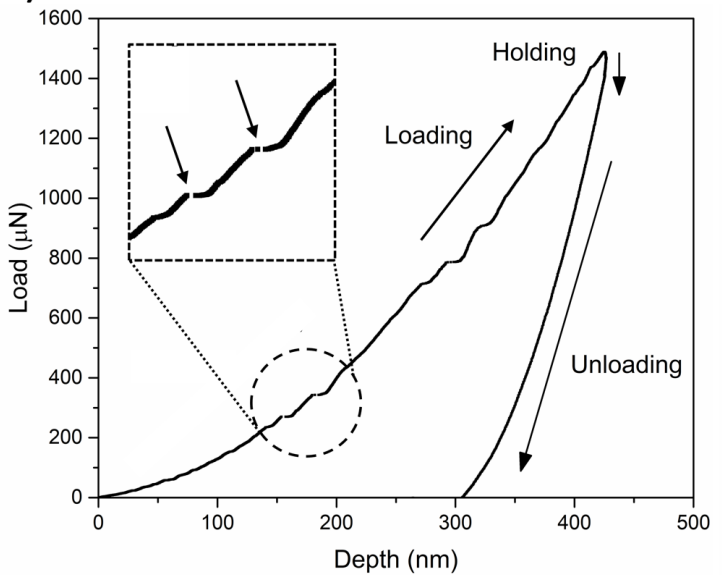

b)

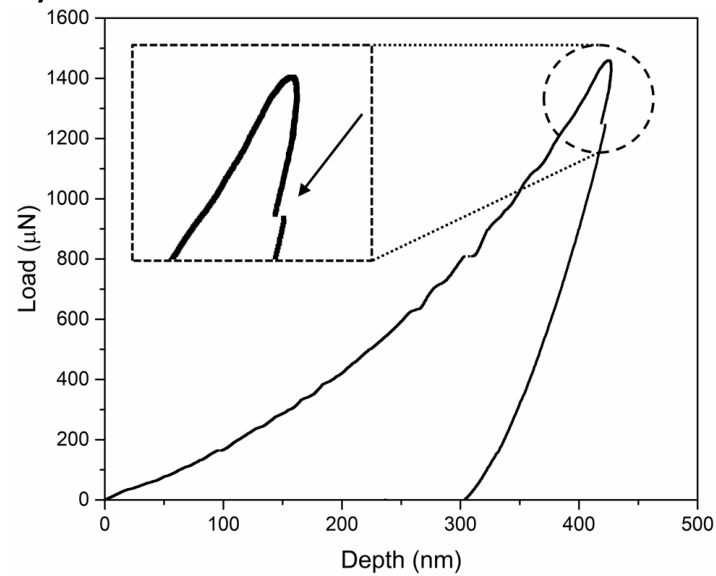

Figure 1: Load-depth curves obtained from nanoindentation on aspirin-I-(100). Indents were carried out up to a maximum depth $h_{M A X}=425 \mathrm{~nm}$. (a) The most common load-depth curves show several pop-ins during the loading section of the indentation process and a smooth bowed unloading section. (b) Some curves exhibited a sudden burst, a pop-in (black arrow), upon unloading.

We performed a number of indents at various in-plane orientations and then used atomic force microscopy (AFM, Multimode 8, Bruker Nano, Santa Barbara CA, USA, using PeakForce QNMmode, in air) to characterise and visualise the surface deformation after each indentation experiment. Figure 2 (a-e) shows height map images of the region surrounding the residual indents. These images were flattened using a $1^{\text {st }}$ order flattening function applying the Nanoscope Analysis software (version 1.8, Bruker) while masking the indented area for the flattening process. The images show in-plane rotated indents from 0 to $60^{\circ}$ every $15^{\circ}$. To aid the analysis, all images have been aligned along the same crystallographic orientations with the aspirin-I b-axis oriented vertically and the c-axis horizontally. This experiment allowed for i) confirmation that the features observed were not due to anomalies with the Berkovich tip (i.e. a defective/damaged tip or contamination on the tip) and ii) assessment of the impact of the inplane orientation of the sample on the resulting indent deformations. The AFM image scale shows light, whiter areas to be elevated whereas dark, blacker areas are depressions. The formation of pile-ups (lighter areas) along some indent edges is observed for all in-plane orientations. The in-plane orientation, however, seems to have an impact on the number of pileups: Figure 2a-d shows pile-ups along two indent edges whilst Figure 2e shows pile-ups occurring at all three edges. Pile-ups correspond to plastic flow near and along the indenter faces, where the material is piling up vertically and the amount of pile-up is related to the hardness/modulus ratio. ${ }^{13}$ 
a) $0^{\circ}$

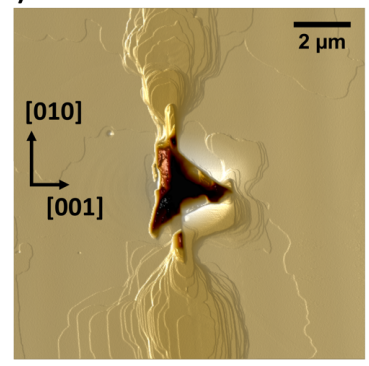

d) $45^{\circ}$

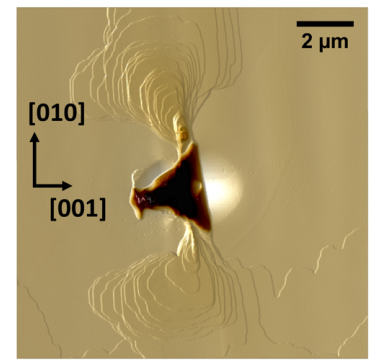

b) $15^{\circ}$

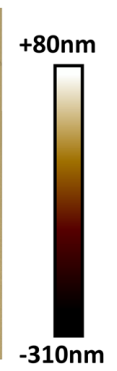

$-310 n m$

e) $60^{\circ}$

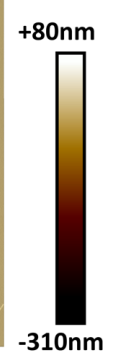

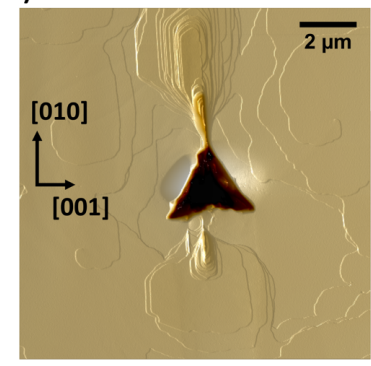

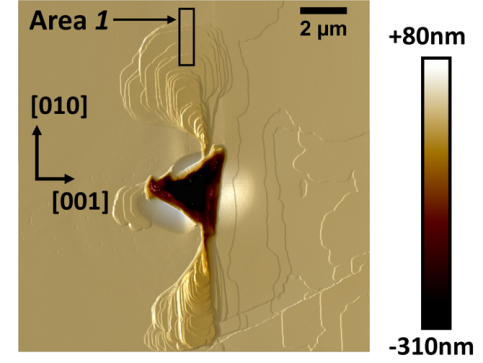

c) $30^{\circ}$

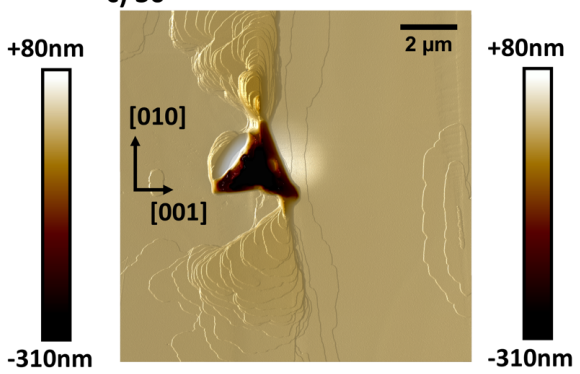

f) Step height analysis, Area 1

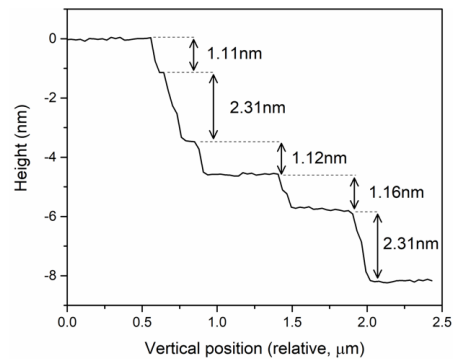

Figure 2: AFM height map images of indents carried out at the surface of a cleaved aspirin-I-(100) at various in-plane orientations of the crystal on the nanoindenter stage (a) $0^{\circ}$ (b) $15^{\circ}$ (c) $30^{\circ}$ (d) $45^{\circ}$ (e) $60^{\circ}$. (f) Step height analysis of the Area 1 delimited by the black rectangle in image (e). The $b$-axis ([010] direction) and the c-axis ([001] direction) are respectively oriented vertically and horizontally in all images.

Above and below each indent, we observe some material steps nucleating from the indents and propagating along the [010] direction, which we refer to as depressed nanoterraces (Figure 2ae). Cleaving the sample (100) surface prior to indentation was essential here to allow for the observation of these depressed nanoterraces since it led to extremely low surface roughness which in turn allowed for AFM images with excellent resolution. In Figure 2f, we are able to visualise the vertical heights of each step in one of these nanoterraces (Area 1 within the black rectangle in Figure 2e). The step height analysis was done using the Step Function within the Nanoscope Analysis software and resulted in averages of the step heights of 1.11, 2.31, 1.12, 1.16 and $2.31 \mathrm{~nm}$. All those step heights closely match an integer value of the lattice parameter $\boldsymbol{a}$ which is $1.14 \mathrm{~nm}$ in aspirin-I. These depressed nanoterraces propagate from the indents prevalently along the [010] direction with their edges well delimited by the (002), (010), (011 ) and (01-2) planes (Figure 3). Especially, the (002) edges seem to dominate. The maximum depth of the depressions ranges from 15 to $80 \mathrm{~nm}$ in areas located right next to the indents up to nearly $1.14 \mathrm{~nm}$ (one $d_{100}$ thick) depth further away from the indents. They are thus rather shallow as compared to the indents' depths $\left(h_{\max }=425 \mathrm{~nm}\right)$. 


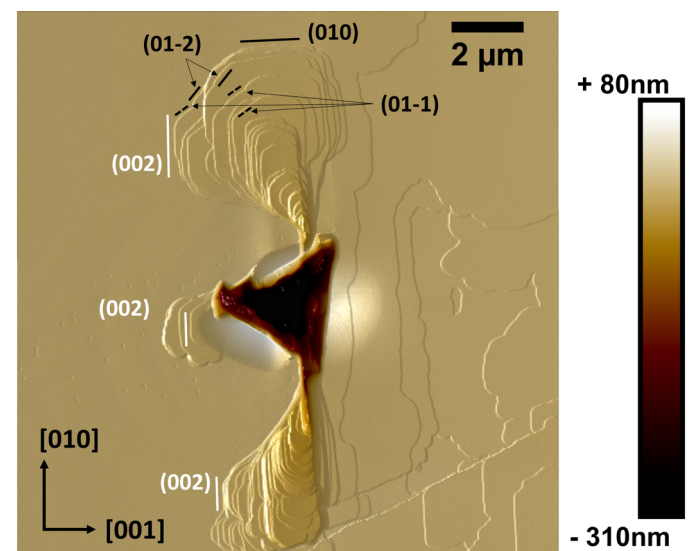

Figure 3: AFM height map image of an indent at the surface of a cleaved aspirin-I-(100). Plastic deformation occurred around the indents (pile-ups) and terraces of one or two times $d_{100}=1.14 \mathrm{~nm}$ formed via spalling fracture of (100) planes which propagated dominantly along [010]. Terraces orientations matched crystallographic planes, in particular (002), (010), (01-1) and (01-2) planes.

There are two plausible mechanisms which could lead to the formation of the depressed nanoterraces observed here in aspirin-I-(100), Figures 2 and 3. First, the nanoterraces could originate from a dislocation motion, which has been reported to result in slip steps with the form of oriented terraces in other materials such as in gold single crystals. ${ }^{14,15}$ The terraces generated via this mechanism, however, are positive and similar in many ways to small amount of pile-up, thus they result in upward steps. In our AFM images, the nanoterraces are below the unindented sample surface, suggesting that the dislocation motion mechanism might not be responsible for this effect. The second plausible mechanism able to generate the negative nanoterraces is spalling fracture, a mechanism by which brittle materials break via a combination of cracks which results in the ejection of chips of the material. Spalling fracture is commonly observed while indenting multi-layered materials (materials with weakly bound layers) such as thin brittle films deposited over a hard substrate ${ }^{11,16}$, muscovite (mica) ${ }^{17}$ or graphite crystals. ${ }^{18}$ Chipping of aspirin single crystals surfaces has already been observed by Olusanmi and coworkers, who carried out impact tests which led to portions of aspirin surfaces being chipped off - leaving surfaces with "staircase-like patterns" somewhat similar to those observed in Figure 2 but at a much larger length scale. ${ }^{19}$ Beyond the images of the nanoterraces, we notice that pop-ins occur upon loading and sometimes upon unloading, during the indentation experiments. These pop-ins upon unloading are strongly indicative of lateral cracking, below and parallel to the surface. The possible association of the pop-ins upon loading with the spalling ejection proposed here explains why their intensity could not be correlated with the interplanar distance $d_{100}$ (from ACSALA $01^{20}$ ). Such correlation between pop-ins and $d_{100}$ would not be expected for pop-ins occurring due to the formation of lateral cracks propagating perpendicular to the indentation direction rather than related to the collapse of the indented layers. The AFM height images indicate height depressions rather than elevations, and the "scallop-shape depressed nanoterraces" observed in aspirin-I (Figure 2) are similar to those reported by Cook and Pharr as a typical result from "shallow lateral cracks", which nucleate not far below the surface and propagate outward. ${ }^{21}$ We note that step-like depressions are also observed right beyond the top-left pile-up in Figure 2c, and in some extent in Figure 2e (bottomleft pile-up), indicating plastic-induced brittleness.

To help visualise and rationalise spalling fracture in aspirin at the molecular level, we performed a crystallographic analysis together with computations of surface attachment 
energies $\left(U_{A T T}\right)$. In Figure 4, a packing view of a (100) step in a depressed nanoterrace in form I aspirin is presented together with two relevant crystallographic planes. The computed attachment energies for those as well as other important families of crystallographic planes are presented in Table 1. Half of the attachment energy of a given $(h k l)$ plane is defined as the energy required to break the intermolecular interactions between a mole of molecules in a $(h k l)$ slice (crystallographic layer) of molecules and the next slice. This is directly analogous to the fracture energy of an ideally brittle material; i.e. the energy of fracture is the energy released by breaking the atomic bonds across the fracture plane minus that required to form the new surfaces.

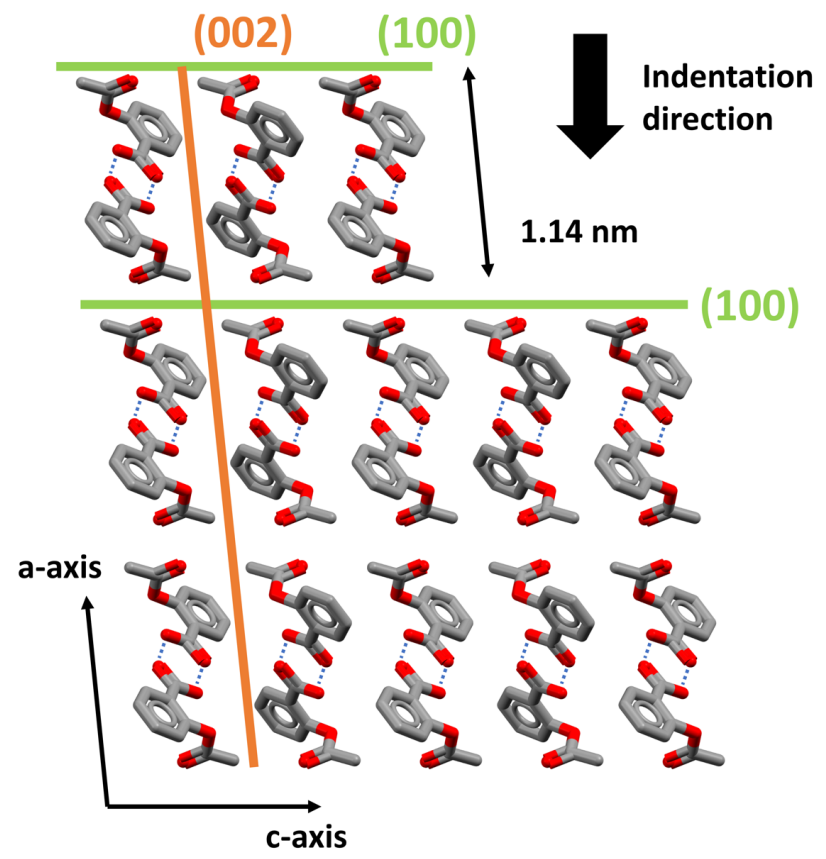

Figure 4: Packing view of aspirin form I along the $b$-axis. A (100) terrace of $1.14 \mathrm{~nm}$ is shown at the molecular level and the (100) and (002) planes are highlighted in green and orange respectively.

Table 1: Attachment energies for the major surfaces observed in the aspirin form I crystals

(ACSALA01).

\begin{tabular}{cccc}
\hline $\begin{array}{c}\{h k\} \\
\text { Family }\end{array}$ & $\begin{array}{c}\text { Number of } \\
\text { planes }\end{array}$ & $\begin{array}{c}U_{\text {ATT }} \\
{[\mathrm{a}](\mathrm{kJ} / \mathrm{mol})}\end{array}$ & $\begin{array}{c}0.5 U_{A T T} \\
{[\mathrm{a}](\mathrm{kJ} / \mathrm{mol})}\end{array}$ \\
\hline$\{100\}$ & 2 & -23.7 & -11.9 \\
\hline$\{002\}$ & 2 & -44.2 & -22.1 \\
\hline$\{110\}$ & 4 & -70.3 & -35.2 \\
\hline$\{011\}$ & 4 & -72.9 & -36.5 \\
\hline$\{10-2\}$ & 2 & -62.5 & -31.3 \\
\hline$\{010\}$ & 2 & -97.6 & -48.8 \\
\hline$\{012\}$ & 4 & -83.6 & -41.8 \\
\hline
\end{tabular}


These calculations reveal that the attachment energy of the $(100)$ plane $(-23.7 \mathrm{~kJ} / \mathrm{mol})$ is much less negative than any of the other planes $(-44.2 \mathrm{~kJ} / \mathrm{mol})$, which gives aspirin this multilayered type of behaviour with much softer plane to plane interactions between (100) planes (along the [100] direction). The lateral cracks thus occur on the (100) planes -the terraces- and can propagate on that plane along the [010] and/or the [001] directions. The AFM images show a preferential propagation along the [010] direction resulting in terraces elongated along [010] but narrow along [001] (Figures 2a-e). Propagation along the [010] requires of more inner step edges dominated by the (002) plane which is, energetically, the second easiest plane to break. In Figure 3, we show that (100) layers are broken dominantly along their respective (002) plane compared to the (010). Almost no terraces nucleated along [001] directly from the indents, however some are visible further away, up and down the indents.

To further understand the inhomogeneous appearance of these terraces along [010] and [001], we computed both the elastic stiffness and compliance constants of aspirin-I (detailed in the Electronic Supplementary Information, ESI) using the COMPASS-II forcefield as implemented in Materials Studio. From these, we calculated and compared the shear moduli corresponding to the aforementioned planes. The shear moduli $\mathrm{G}_{12}$ and $\mathrm{G}_{13}$ were calculated to be equal to $1.81 \mathrm{GPa}$ and $1.11 \mathrm{GPa}$ respectively. In other words, for a shearing force along the a-axis, the resistance to shear is $63 \%$ higher for the (010) plane than for the (002) plane; this is further confirmed by the significantly higher energy of attachment $U_{A T T}$ for $(010)$ than for (002) (see Table 1). The low shear resistance of the (002) plane matches our observations of (002) terraces above and below the indents as shown in Figure 3 and hence further explains the preferential propagation along [010]. These observations once more emphasise the strong anisotropy of mechanical properties in molecular single crystals.

In conclusion, we have shown evidences that spalling fracture occurs in a molecular crystal upon nanoindentation. We believe, to the best of our knowledge, that this is the first report of such a phenomenon in molecular crystals at room temperature. An analysis of the crystallographic directions in aspirin-I combined with some computations revealed a strongly anisotropic mechanical behaviour and enabled us to rationalise the direction of propagation of the cracks and consequently the spalling fracture. We note that we were unable to observe the ejected nanochips of aspirin-I since at room temperature these nanoscale debris are unlikely to remain intact, most likely melting soon after ejection. This fracture behaviour at the nanoscale can be closely related to similar fracture behaviour at larger length scales (the nanochipping upon nanoindentation observed by us can be related to the microchipping observed in aspirin crystals after impact tests ${ }^{19}$ ). A better understanding of the mechanisms of fracture in molecular crystals is a necessity in order to better design processes such as milling which are very common pharmaceutical manufacturing.

\section{Experimental Section}

Experiments were carried out using a Hysitron TI 950 TriboIndenter (Bruker Nano, Minneapolis MN, USA) equipped with a diamond Berkovich tip. Indents were performed on form I aspirin single crystals where the crystal shape clearly matched that identified during a face indexing reported previously. ${ }^{8}$ This enabled the identification of the $(a, b, c)$ crystallographic axes during indentation. Samples were cleaved along their dominant (100) face using adhesive tape. Indentations were conducted in displacement-controlled mode using a 
trapezoid load function with 5 second loading, 5 second hold and 5 second unloading segments. The distance between individual indents was set to $4 \mu \mathrm{m}$. Five indents were carried out, followed by consecutive rotations of 15 degrees, with five indentation events made for each angle. Imaging of the samples was carried out by atomic force microscopy (AFM, Multimode 8, Bruker Nano, Santa Barbara CA, USA) using PeakForce QNMmode, in air, with a TESPV2 probe $(k=42 \mathrm{~N} / \mathrm{m})$. AFM imaging was performed within 6 hours of the indents being produced. Materials Studio 2019 (Dassault Systèmes, BIOVIA) ${ }^{22}$ was used to compute the mechanical properties, including the elastic stiffness and compliance constants, and the attachment energies of aspirin form-I with the COMPASS-II forcefield. ${ }^{23}$

Supporting Information Extended experimental details and computed and stiffness and compliance matrixes.

\section{Acknowledgements}

BG thanks Roche and the University of Manchester for financial support, Sin Kim Tang for her help crystallising $p$ TA and $p$ BOBA crystals, and the staff in the Department of Chemistry XRD Facility in the School of Natural Sciences at the University of Manchester for their assistance. AJCC thanks the Royal Society for an industry Fellowship in AstraZeneca. This work was supported by the Henry Royce Institute for Advanced Materials, funded through EPSRC grants EP/S009493/1, EP/R00661X/1, EP/P025021/1 and EP/P025498/1. The authors thank Dr Matthias Lauer and Pirmin Hidber for helpful discussions.

\section{References}

(1) Ramamurty, U.; Jang, J. Il. Nanoindentation for Probing the Mechanical Behavior of Molecular Crystals-a Review of the Technique and How to Use It. CrystEngComm 2014, 16, 12-23.

(2) Wang, C.; Sun, C. C. The Landscape of Mechanical Properties of Molecular Crystals. CrystEngComm 2020, 20, 1149-1153.

(3) Reddy, C. M.; Rama Krishna, G.; Ghosh, S. Mechanical Properties of Molecular Crystals - Applications to Crystal Engineering. CrystEngComm 2010, 12, 2296-2314.

(4) Krishna, G. R.; Shi, L.; Bag, P. P.; Sun, C. C.; Reddy, C. M. Correlation among Crystal Structure, Mechanical Behavior, and Tabletability in the Co-Crystals of Vanillin Isomers. Cryst. Growth Des. 2015, 15, 1827-1832.

(5) Saha, S.; Desiraju, G. R. Trimorphs of 4-Bromophenyl 4-Bromobenzoate. Elastic, Brittle, Plastic. Chem. Commun. 2018, 54, 6348-6351.

(6) Reddy, C. M. Mechanical Deformation Chemistry of Crystals: Designing Mechanical Performance. In Molecules to Crystals to Powders: Understanding Structure Versus Function; Springer: Enrice, Sicily, Italy, 2015; pp 425-435.

(7) Varughese, S.; Kiran, M. S. R. N.; Solanko, K. A.; Bond, A. D.; Ramamurty, U.; Desiraju, G. R. Interaction Anisotropy and Shear Instability of Aspirin Polymorphs Established by Nanoindentation. Chem. Sci. 2011, 2, 2236-2242.

(8) Gabriele, B. P. A.; Williams, C. J.; Lauer, M. E.; Derby, B.; Cruz-Cabeza, A. J. Nanoindentation of Molecular Crystals: Lessons Learned from Aspirin. Cryst. Growth Des. 2020, 20, 5956-5966.

(9) Bor, B.; Giuntini, D.; Domènech, B.; Swain, M. V.; Schneider, G. A. NanoindentationBased Study of the Mechanical Behavior of Bulk Supercrystalline Ceramic-Organic Nanocomposites. J. Eur. Ceram. Soc. 2019, 39, 3247-3256.

(10) Pharr, G. M. The Anomalous Behavior of Silicon during Nanoindentation. In Thin 
Films: Stresses and Mechanical Properties III. Materials Research Society Symposium Proceedings, Volume 239; Boston, Massachusetts, 1992; pp 301-312.

(11) Li, X.; Bhushan, B. Measurement of Fracture Toughness of Ultra-Thin Amorphous Carbon Films. Thin Solid Films 1998, 315, 214-221.

(12) Fsicher-Cripps, A. Other Techniques in Nanoindentation - 9.4 Fracture Toughness. In Nanoindentation; 2011; pp 167-169.

(13) Bolshakov, A.; Pharr, G. M. Influences of Pileup on the Measurement of Mechanical Properties by Load and Depth Sensing Indentation Techniques. J. Mater. Res. 1998, 13, 1049-1058.

(14) Carrasco, E.; Rodríguez de la Fuente, O.; González, A.; Rojo, M. Dislocation Cross Slip and Formation of Terraces around Nanoindentations in $\mathrm{Au}(001)$. Phys. Rev. B Condens. Matter Mater. Phys. 2003, 68, 1-4.

(15) Asenjo, A.; Jaafar, M.; Carrasco, E.; Rojo, J. M. Dislocation Mechanisms in the First Stage of Plasticity of Nanoindented Au(111) Surfaces. Phys. Rev. B - Condens. Matter Mater. Phys. 2006, 73, 1-7.

(16) Fu, K.; Tang, Y.; Chang, L. Toughness Assessment and Fracture Mechanism of Brittle Thin Films Under Nano-Indentation. In Fracture Mechanics - Properties, Patterns and Behaviours; 2016; Vol. i, pp 121-144.

(17) Pant, R.; Hu, L.; Zhang, G. Anisotropy of Mica Probed by Nanoindentation. In Multiphysical Testing of Soils and Shales; Laloui, L., Ferrari, A., Eds.; Springer Berlin Heidelberg: Berlin, Heidelberg, 2013; pp 239-245.

(18) Skinner, J.; Gane, N. The Deformation and Twinning of Graphite Crystals under a Point Load. Philos. Mag. 1973, 28, 827-837.

(19) Olusanmi, D.; Roberts, K. J.; Ghadiri, M.; Ding, Y. The Breakage Behaviour of Aspirin under Quasi-Static Indentation and Single Particle Impact Loading: Effect of Crystallographic Anisotropy. Int. J. Pharm. 2011, 411, 49-63.

(20) Yesook, K.; Katsunosuke, M.; Tooru, T.; Kenji, O. Structure Redetermination and Packing Analysis of Aspirin Crystal. Chem. Pharm. Bull. 1984, 33, 2641-2647.

(21) Cook, R. F.; Pharr, G. M. Direct Observation and Analysis of Indentation Cracking in Glasses and Ceramics. J. Am. Ceram. Soc. 1990, 73, 787-817.

(22) Dassault Systèmes BIOVIA, Materials Studio, Release 2019, San Diego: Dassault Systèmes. 2019.

(23) Sun, H.; Jin, Z.; Yang, C.; Akkermans, R. L. C.; Robertson, S. H.; Spenley, N. A.; Miller, S.; Todd, S. M. COMPASS II: Extended Coverage for Polymer and Drug-like Molecule Databases. J. Mol. Model. 2016, 22, 1-10. 


\section{For Table of Contents Use Only:}

\section{Brittle Behaviour in Aspirin Crystals: Evidence of Spalling Fracture}

Benjamin P. A. Gabriele ${ }^{\mathrm{a}}$, Craig J. Williams ${ }^{\mathrm{b}}$, Douglas Stauffer ${ }^{\mathrm{c}}$, Brian Derby ${ }^{\mathrm{b}}$, Aurora J. Cruz-Cabeza*

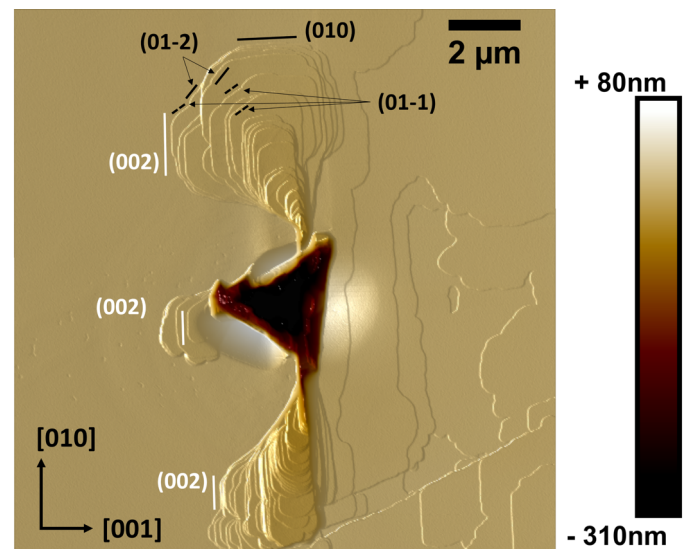

Nanoindentation experiments on the (100) face of aspirin form I reveal brittle behaviour through spalling fracture. 